\title{
The temporal crescent syndrome
}

\author{
Khalid Ali
}

\section{Correspondence to}

Dr Khalid Ali, Stroke Unit, Royal Gwent Hospital, Newport,

Gwent NP20 2UB, UK ;

khalid_w2003@yahoo.co.uk

Accepted 27 October 2014

Published Online First

21 November 2014
CrossMark

To cite: Ali K. Pract Neurol 2015:15:53-55.

\section{ABSTRACT}

Retro-chiasmal lesions almost always give rise to homonymous field defects with only one exception. The nasal visual field extends to $60 \%$ of the horizon, whereas the temporal field extends to a further $30^{\circ}-40^{\circ}$ beyond that; this part of the visual field is represented on the contralateral anterior parieto-occipital sulcus. A lesion in this area will give rise to monocular visual field defect affecting the contralateral eye. This is called the temporal crescent or the half moon syndrome. In this case report, a woman presented with seizures secondary to haemorrhagic infarction of the anterior part of the parieto-occipital sulcus. She later presented with right-sided visual disturbance; her examination confirmed temporal crescent syndrome. I explain the pathophysiology of this rare neurological syndrome in this report.

\section{CASE REPORT}

A 78-year-old woman presented to hospital with two generalised tonic-clonic seizures. She was found at home acutely confused and with right-sided weakness. She had history of bowel pseudo-

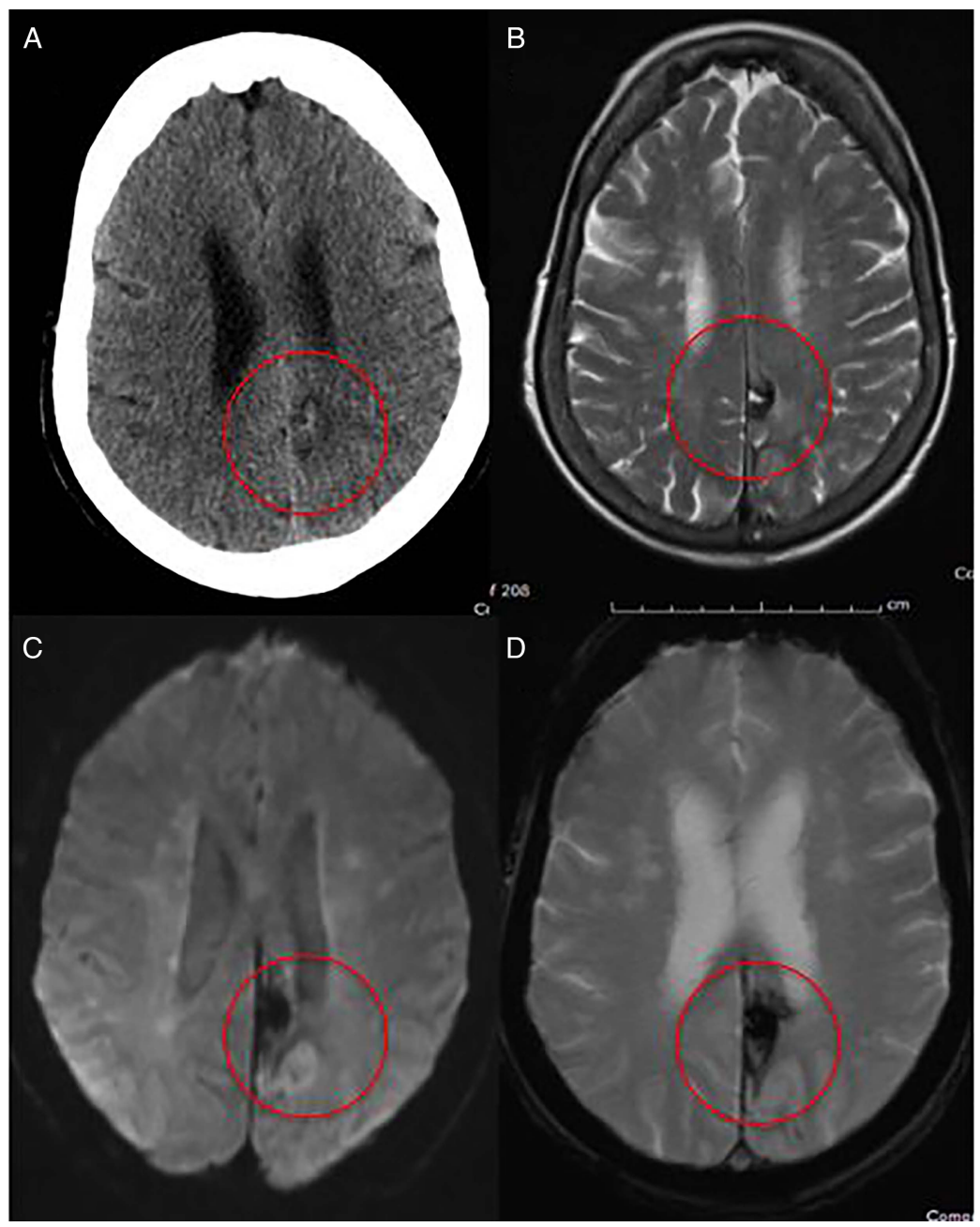

Figure 1 (A) CT scan of head showing a hyperdense lesion at the left parieto-occipital sulcus, indicating haemorrhage surrounded by a hypodense (infarcted) area. (B) MR scan of brain (T2-weighted) showing flow void in the same area, indicating haemosiderin deposition. (C) MR scan of brain (diffusion-weighted image) showing an area of restricted diffusion, indicating acute ischaemia. (D) MR scan of brain (gradient echo sequence) indicating haemosiderin deposition from bleeding. 
obstruction and pulmonary embolism 6 months before, treated with warfarin. She drank little alcohol and did not smoke. In hospital, she presented with right-sided visual disturbance.

Her general examination was normal. She had normal visual acuity. There was clear crescent-shaped restriction of the temporal aspect of right eye's visual field on a confrontation test, whereas her left eye's visual field was normal. Pupillary reflexes, colour vision and fundal examination were normal. Eye movements were normal. The remaining neurological examination was normal.

Her full blood count, urea and electrolytes and serum C reactive protein were normal; CT scan of her head showed a hyperdense lesion surrounded by hypodensity in the anterior part of the left parieto-occipital sulcus (figure 1A). MR scan of brain showed a circular area of flow void surrounded by increased signal in the same area. Diffusion-weighted MRI suggested an area of restricted diffusion surrounding that. The gradient echo sequence suggested haemosiderin deposit at the area of the flow void suggesting an area of haemorrhagic transformation (figure 1B-D). Her ECG showed new onset of atrial fibrillation.

She recovered well and spent 14 days in a stroke rehabilitation unit before discharge. Three weeks later, she was readmitted with visual disturbance of flashing lights at the extreme right visual field associated with mild headache. Repeat CT scan of head showed no new abnormality. We made a diagnosis of occipital epilepsy and she started levetiracetam.

\section{DISCUSSION}

The rule of thumb in visual field assessment is that retrochiasmal lesions cause homonymous defects. The only exception is that of the 'missing temporal crescent' or 'half moon syndrome'. ${ }^{1}$ The nasal visual field extends to $60 \%$ of the horizon, whereas the temporal field extends to $100 \%$. Thus, $30 \%-40 \%$ of the peripheral temporal visual field is unpaired; this part of the visual field, therefore, has unilateral representation on the contralateral visual cortex (figures 2 and 3 ). Damage in this area of the visual cortex leads to a contralateral temporal crescent. ${ }^{23}$

Gordon Holmes produced the first retinotopic representation of the visual field on the human occipital cortex. He and others studied visual field deficits in patients with wartime penetrating injuries into the occipital cortex. He showed that the upper visual field is represented by the inferior calcarine cortex, whereas the lower visual field maps to the superior calcarine cortex. The central visual field is represented posteriorly and the peripheral visual field, including the temporal crescent, is represented more anteriorly. Holmes' map suggests that the central $15^{\circ}$ of vision (ie, the macula) covers $25 \%$ of the entire visual cortex. ${ }^{4}$ More recently, Horton and $\mathrm{Hoyt}^{5}$ used

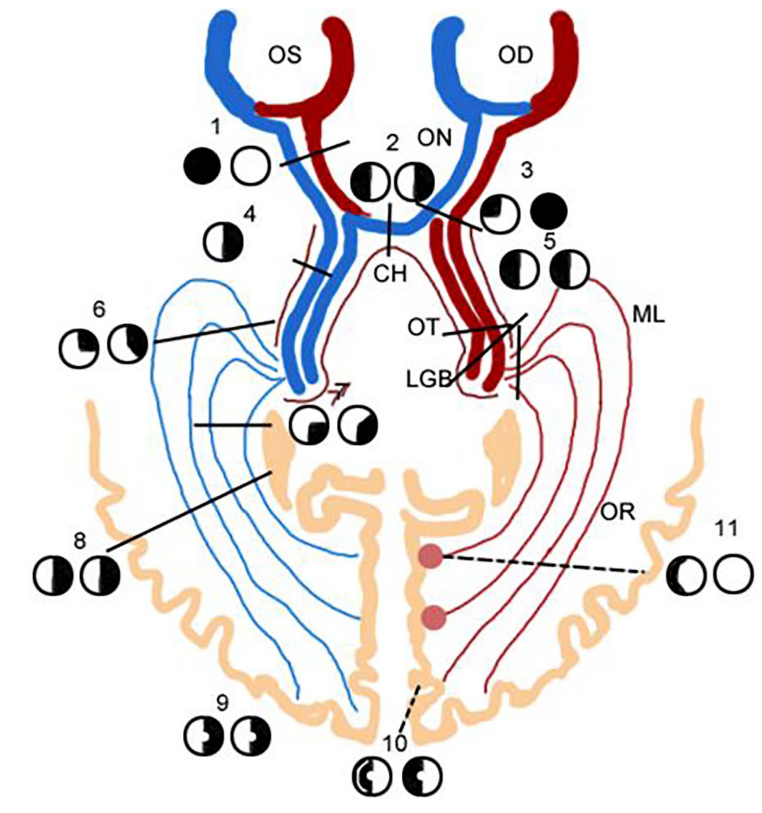

Figure 2 Schematic representation of the visual pathway and the location of lesion leading to the temporal crescent syndrome. The numbers on the scheme represent the various patterns of visual field defects from lesions into the various components of the visual pathway, number 11 represents the site of the lesion leading to temporal crescent syndrome. OS, left eye; OD, right eye; ON, optic nerve; $\mathrm{CH}$, chiasm; OT, optic tract; LGB, lateral geniculate body; ML, Meyer's loop; OR, optic radiation.

Holmes' map to correlate visual field defects with MR scan findings; they concluded that the central $15^{\circ}$ of central vision occupies $70 \%$ of the entire visual cortex. The temporal crescent is represented by $10 \%$ of the visual cortex, which is situated anteriorly. ${ }^{56}$

This patient's MRI suggested infarction with haemorrhagic transformation affecting the anterior part of the parieto-occipital sulcus at its junction with the calcarine fissure.

Acute symptomatic seizures and epilepsy each occur in stroke, particularly with cortical involvement. ${ }^{7} 8$ Intracerebral haemorrhage, whether primary or

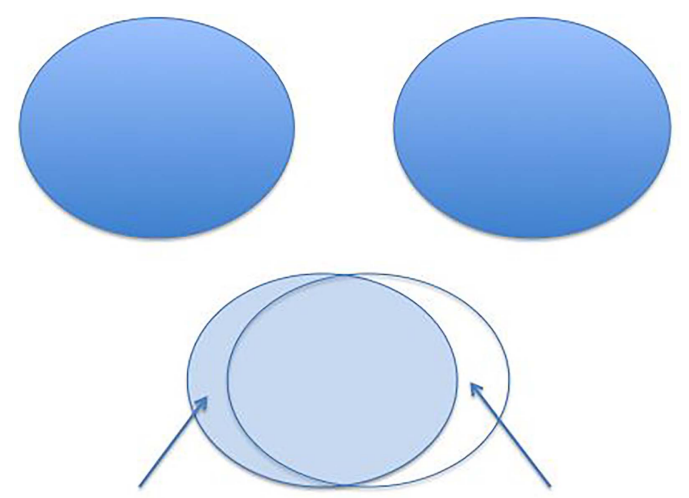

Temporal crescent/ half moon syndrome

Figure 3 Schematic representation of the visual field. 


\section{Learning points}

- Retro-chiasmal lesions normally cause homonymous visual field defects; the only exception is the 'temporal crescent' or 'half moon syndrome', caused by a lesion in the anterior part of the contralateral parieto-occipital sulcus.

- The most nasal retinal fibers have unpaired cortical representation, which is situated in the most anterior part of the contralateral parieto-occipital sulcus.

secondary to ischaemic stroke, increases the risk of poststroke epilepsy. ${ }^{7} 8$ Embolic stroke-as in our patient-also increases the risk of epilepsy. ${ }^{8}$

Clinicians need to be aware of this syndrome because of its localising and diagnostic importance.

Competing interests None.

Patient consent Obtained.
Provenance and peer review Not commissioned; externally peer reviewed. This paper was reviewed by Mark Lawden, Leicester, UK.

\section{REFERENCES}

1 Cooper SA, Metcalfe RA. Assess and interpret the visual fields at the bedside. Pract Neurol 2009;9:324-34.

2 Landau K, Wichmann W, Valavanis A. The missing temporal crescent. Am J Ophthalmol 1995;119:345-9.

3 Benton S, Levy I, Swash M. Vision in the temporal crescent in occipital infarction. Brain 1980;103:83-97.

4 Wong AM, Sharpe JA. Representation of the visual field in the human occipital cortex: a magnetic resonance imaging and perimetric correlation. Arch Ophthalmol 1999;117:208-17.

5 Horton JC, Hoyt WF. The representation of the visual field in human striate cortex. A revision of the classic Holmes map. Arch Ophthalmol 1991;109:816-24.

6 Chavis PS, al-Hazmi A, Clunie D, et al. Temporal crescent syndrome with magnetic resonance correlation. J Neuroophthalmol 1997;17:151-5.

7 Powell R, McLauchlan DJ. Acute symptomatic seizures. Pract Neurol 2012;12:154-65.

8 Myint PK, Staufenberg EFA, Sabanathan K. Post-stroke seizure and post-stroke epilepsy. Postgrad Med J 2006;82:568-72. 